\title{
Progress towards rabies elimination from Pemba Island, Southern Tanzania
}

\author{
Kennedy S. Lushasi ${ }^{\star 1,2}$, Sarah Cleaveland ${ }^{2}$, Joel J. Changalucha ${ }^{1,2}$, Daniel Haydon ${ }^{2}$, \\ Rudovick Kazwala ${ }^{3}$, Tiziana Lembo ${ }^{2}$, Msanif Masoud ${ }^{4}$, Mathew Maziku5, \\ Geofrey Mchau ${ }^{6}$, Zacharia Mtema', Kassim Omar ${ }^{7}$, Sambo Maganga ${ }^{1,2}$, \\ Kristyna Rysava ${ }^{2}$ and Katie Hampson ${ }^{1,2}$
}

${ }^{1}$ Environmental Health and Ecological Sciences, Ifakara Health Institute, Ifakara, Tanzania, United Republic of; ${ }^{2}$ Glasgow University, Glasgow, United Kingdom; ${ }^{3}$ Sokoine University of Agriculture, Morogoro, Tanzania, United Republic of; ${ }^{4}$ Ministry of Health, Zanzibar, Pemba, Tanzania, United Republic of; ${ }^{5}$ Ministry of Livestock and Fisheries Development, Dar es Salaam, Tanzania, United Republic of; ${ }^{6}$ Ministry of Health and Social Welfare, Dar es Salaam, Tanzania, United Republic of; ${ }^{7}$ Ministry of Livestock and Fisheries,

Zanzibar, Pemba, Tanzania, United Republic of

\section{Objective}

Using active surveillance approaches to investigate the transmission dynamics of rabies on Pemba Island and across Southern Tanzania, whilst a large-scale dog vaccination program was underway ${ }^{1}$, to gain a greater understanding of the dynamics of infection as the disease is driven towards elimination.

\section{Introduction}

Rabies is endemic in Tanzania and has circulated on Pemba Island since the late 1990s. In 2010, an elimination programme was initiated in Southern Tanzania to demonstrate that human rabies deaths can be eliminated through mass dog vaccinations. We used active surveillance approaches ${ }^{2}$ to investigate the dynamics of rabies across the area where this programme was implemented.

\section{Methods}

Government census data and post-vaccination transects were used to estimate the dog population and coverages achieved by vaccination campaigns. Routine surveillance of animal bite injuries using a mobile phone-based surveillance system ${ }^{3}$ and active contact tracing were used to identify animal rabies cases and human exposures. Epidemic trees were constructed using spatiotemporal distances between cases and used to estimate the effective reproduction number $\left(\mathrm{R}_{\mathrm{e}}\right)$. We examined factors affecting rabies incidence and transmission using generalized linear mixed models.

\section{Results}

We estimated a small dog population of 4095 and low dog:human ratio on Pemba (1:105). Overall island-wide vaccination coverage increased from $16.8 \%$ in 2011 to $68.2 \%$ in 2014 . We found a further 48 human exposures (343\%), who either were not reported or did not obtain post exposure prophylaxes (PEP). Routine surveillance was found to detect less than $10 \%(\sim 8.75 \%)$. There was a rapid decline in cases detected on Pemba, from 42 before mass dog vaccinations were implemented in 2011, to 2 cases in 2014 (Figures 1). Since May 2014 , no rabies cases have been detected. Similarly, $\mathrm{R}_{\mathrm{e}}$ declined from 1.02 to 0 and a significant relationship was found with rabies cases decreasing with increasing vaccination coverage ( $p=0.013$, Figure 2$)$. Across seven other districts on the Tanzanian mainland we also observed major declines in rabies cases with very few cases of rabies in dogs detected in 2016 (Figure 3).

\section{Conclusions}

We conclude that rabies has been eliminated from domestic dog populations on Pemba over the five years since vaccination campaigns have been implemented. Continued surveillance and investigations of any bite incidents are therefore needed to ensure any subsequent incursions are controlled and freedom from rabies is maintained.
On the Tanzanian mainland, it has taken longer to control rabies, however trajectories look promising with several districts close to eliminating the disease. However, detection of some wildlife cases in the last 12 months in these districts indicates the need to further investigate remaining foci and the role of wildlife in maintenance.

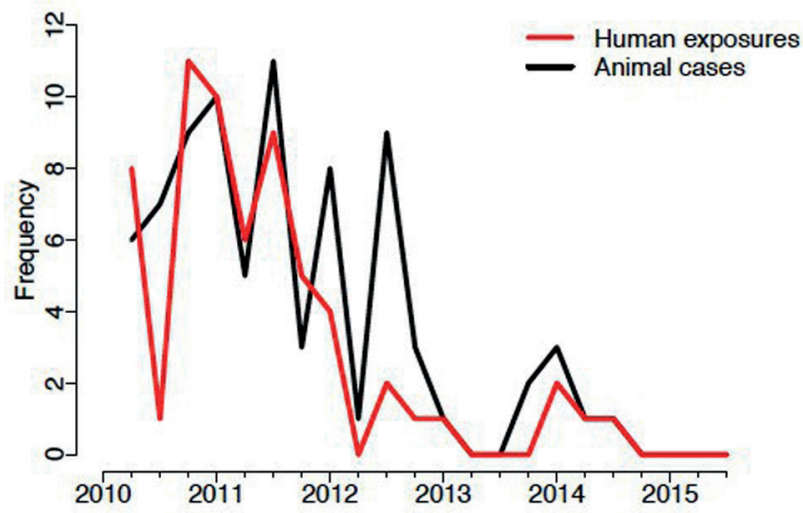

Suspect rabies cases in animals (black) and human exposures (red) on Pemba Island, from 2010 to 2015

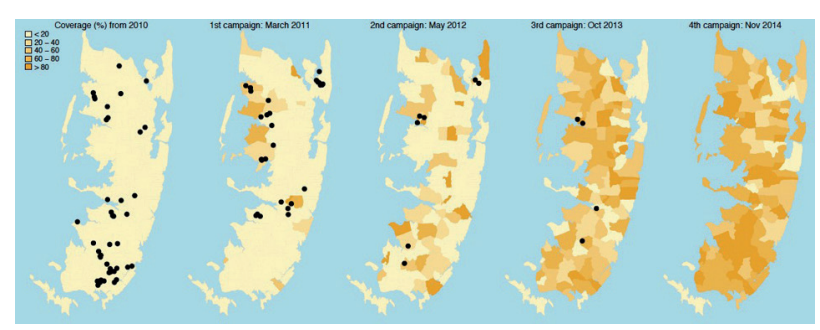

Estimated vaccination coverage of the dog population (shading) in villages on Pemba, and suspected animal rabies cases (dots) since 2010 following each vaccination campaign from 2011 to date. Darker shading corresponds to higher vaccination coverage 

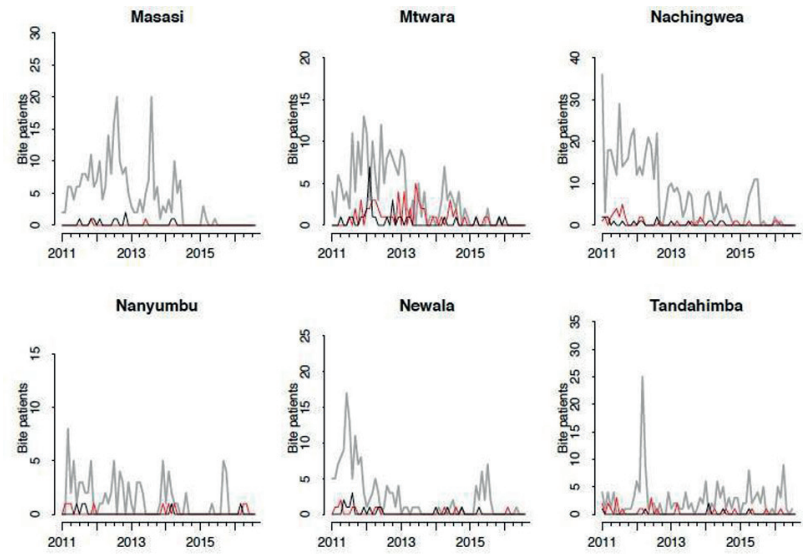

Trajectories of all bite injuries reported to clinics through routine mobile phone-based surveillance (grey) and of rabies cases in dogs (black) and wildlife (red) detected through contact tracing

\section{Keywords}

Canine rabies; elimination; vaccination; dogs; Pemba Island

\section{Acknowledgments}

We are grateful for the support provided by the staff of the Animal and Health Departments of Pemba and to the Tanzania Ministries of Health and Social Welfare, and of Livestock and Fisheries Development, WHO Country Office, and the Bill and Melinda Gates Foundation for support. This work was funded by the Wellcome Trust and UBS Optimus Foundation.

\section{References}

1 WHO. The sixth meeting of the International Coordinating Group (ICG) of the Bill \& Melinda Gates foundation-World Health Organization project on eliminating human and dog rabies. (Durban, South Africa, 2014).

2 Hampson, K. et al. Transmission Dynamics and Prospects for the Elimination of Canine Rabies. Plos Biology 7, e1000053, doi:10.1371/ journal.pbio.1000053 (2009).

3 Mtema, Z. et al. Mobile Phones As Surveillance Tools: Implementing and Evaluating a Large-Scale Intersectoral Surveillance System for Rabies in Tanzania. PLoS Med 13, doi:10.1371/journal.pmed.1002002 (2016).

\section{*Kennedy S. Lushasi}

E-mail: klushasi@ihi.or.tz 